\title{
Detection of Omental Recurrence of Colorectal Cancer by FDG-PET/CT
}

\author{
MM RAHMAN
}

A 48 year old lady underwent anterior resection of rectosigmoid colon for a tumour in the upper rectum in September 2011.High ligation of the inferior mesenteric artery and total mesorectal excision done during the operative procedure. Histopathology report revealed moderately differentiated adenocarcinoma. All ends of the colon were tumour free. There was no evidence of lymph node involvement. She received six cycle chemotherapy containing Oxaliplatin and capacetabine afterwards. She was running well, enjoying a healthy family life after the treatment. Dietary and bowel habit was normal. She was under strict follow up with S CEA level, USG examination of the abdomen and colonoscopy every six months after initial treatment. All were within normal limit. In January 2015, she developed lower abdominal pain, but bowel habit was normal, serum CEA level was $50 \mathrm{ng} / \mathrm{ml}$, colonoscopy, ultrasonography was normal, only a lump found adhered with parietal wall which was detected in CT scan. But PET/CT examination revealed multiple high FDG uptake suggesting recurrences in the abdominal cavity in the area of lower abdomen, not related with gut in addition to the parietal lump which was earlier detected by CT scan. After laparotomy, along with a lump adhered to parietal wall on the right side, there was multiple seedlings on the omentum matching with the PET/CT findings . Excision of the lump along with omentectomy done. Histopathological analysis yielded a diagnosis of metastatic adenocarcinoma from the omental lesion.

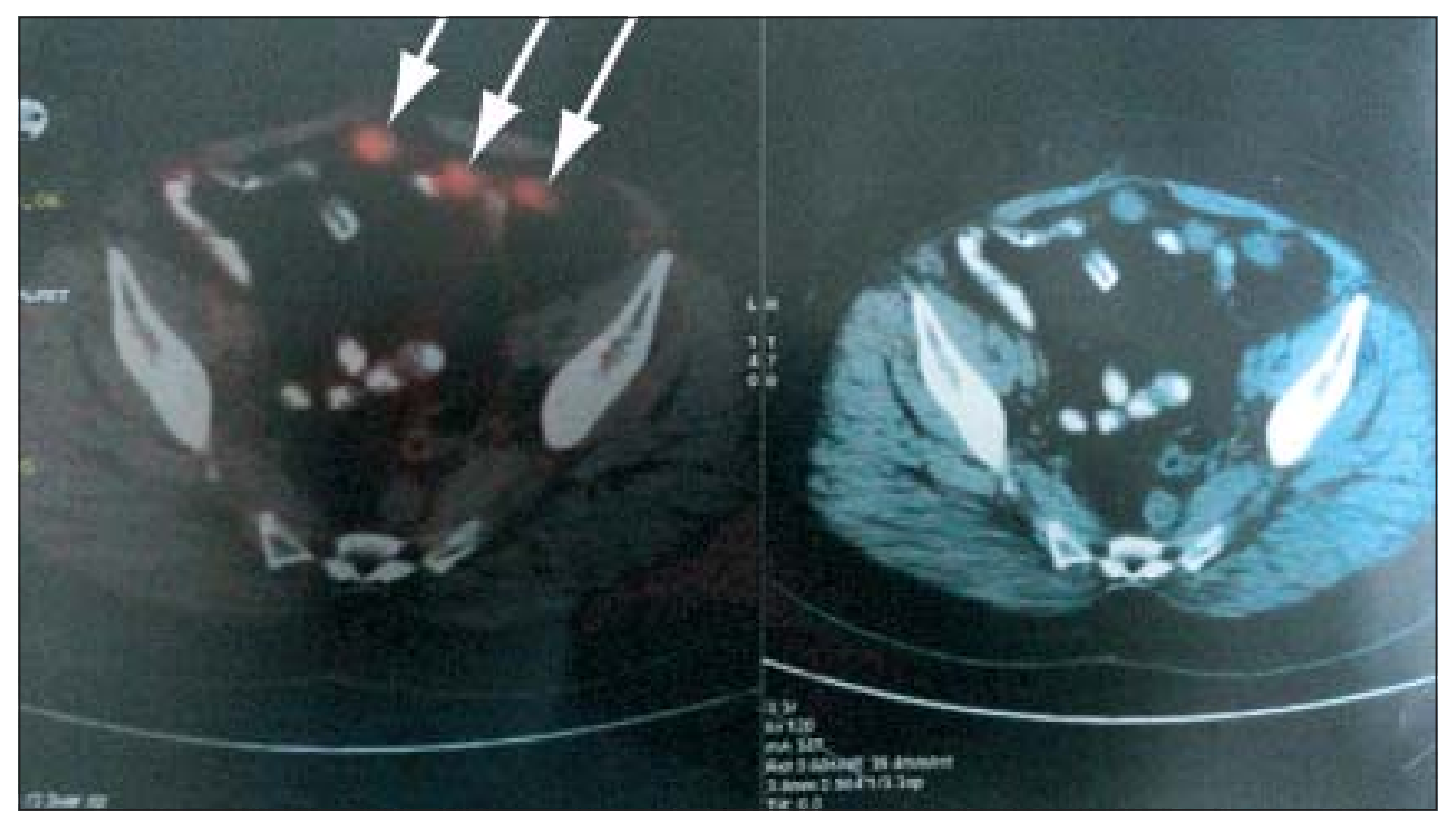

Fig.-1: Marked areas show increased FDG uptake in a PET/CT Scan suggesting metastatic deposits

Address of Correspondence: Prof. Md. Mizanur Rahman Professor and Head, Department of Surgical Oncology, National Institute of Cancer Research and Hospital, Mohakhali, Dhaka, Email: miizannicrh@gmail.com 
Recurrence develops within 3 years after radical surgery for co-lon cancer in $83.6 \%$ of recurrent colon cancer cases and is very rarely detected after 5 years (3.6\% of cases $)^{1}$. Therefore, regular follow-up with various diagnostic modalities until 5 years after surgery is a reasonable strategy to detect recurrent disease. The commonly used imaging modalities in such cases include ultrasonography, computed tomography (CT), and mag-netic resonance imaging (MRI). However, ${ }^{18} \mathrm{~F}$ fluorodeoxyglucose glucose-positron emission tomography (FDG-PET) is more ef-fective in determining the presence of malignancy, especially in cases of colorectal cancer (CRC) ${ }^{2}$.

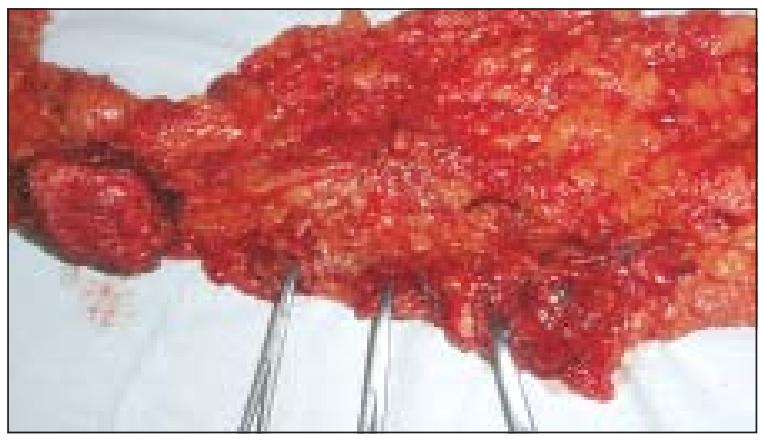

Fig.-2: Post operative view of greater omentun shows three metastatic seedlings marked by artery forceps

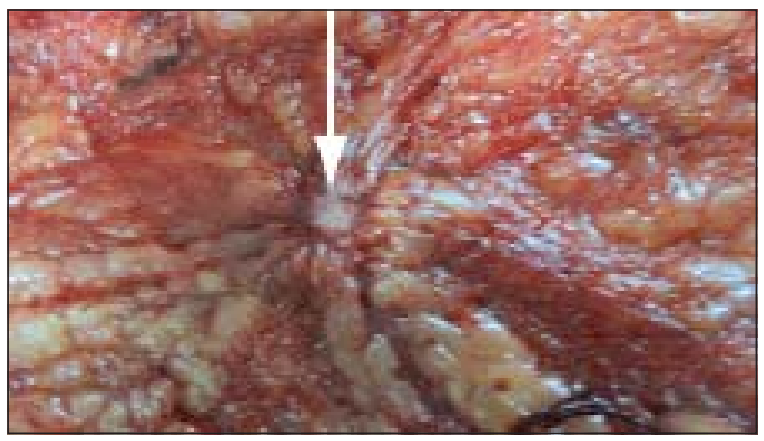

Fig.-3: Close view of the metastatic seedling in the omentum-marked by the arrow.

Locoregional pelvic recurrence and liver metastases are the major sites of relapse after resection of CRC. Recurrence occurs in one third of patients in the first 2 years after resection. Patterns of recurrence are different in colon cancer compared with rectal cancer. Local recurrence is more common in patients with rectal rather than colon cancer ranging from $7 \%$ to $33 \%$ and $1-19 \%$, respectively ${ }^{3}$,

Detection of recurrent lesions usually involves serum tumor marker level measurement, ultrasonography, CT, and MRI. In addition, the efficacy of FDG-PET has been confirmed $^{4}$. Luboldt et al. reported that FDG-PET/CT provided promising accuracy for colorectal mass detection and that, in all carci-nomas and adenomas with high-grade dysplasia ${ }^{5}$. A review by Visioni and Kim stated that the sen-sitivity and specificity of PETCT in detecting CRC recurrence were 89-95\% and 83$92 \%$, respectively ${ }^{6}$. One of the stud-ies evaluated in that review ${ }^{7}$ reported a positive predic-tive value of $96.4 \%$ and a negative predictive value of $76.9 \%$ for the diagnosis of CRC recurrence by PET-CT. So PET/CT is a useful tool for the diagnosis of recurrence of colorectal cancer even in a smaller size within omentum and peritoneum. which are missed in traditional CT scan examination.. After that appropriate measures like radical surgery improves the total survival of the patient.

\section{References:}

1. Kobayashi H, Mochizuki H, Sugihara $\mathrm{K}$ et al: Characteristics of recurrence and surveillance tools after curative resection for colorectal cancer: A mul-ticenter study. Surgery, 2007; 141(1): 67-75.

2. Takashi Orii B Motohiro Okumura B Masaki Yoshimura B Hiroe Kitahara B Yukihiko Karasawa .Am J Case Rep, 2015; 16: 149-152

3. Owen J. O’Connor, Shanaugh McDermott, James Slattery, Dushyant Sahani,andMichael A. Blake. The Use of PET$\mathrm{CT}$ in the Assessment of Patients with Colorectal Carcinoma. Internat J Surg Oncol 2011 http://dx.doi.org/ 10.1155/2011/846512.

4. Sarikaya I, Bloomston M, Povoski SP et al: FDG-PET scan in patients with clinically and/or radiologically suspicious colorectal recurrence but normal CEA. World J Surg Oncol, 2007; 5: 64

5. Luboldt W, Volker T, Wiedemann B et al: Detection of relevant colonic neo-plasms with PET/CT: promising accuracy with minimal CT dose and a stan-dardized PET cut-off. Eur Radiol, 2010; 20(9): 2274-85

6. Visioni A, Kim J: Positron Emission Tomography (PET) for benign and ma-lignant disease. Surg Clin North Am, 2011; 91(1): 249-66

7. Chen LB, Tong JL, Song HZ et al: 18F-DG PET/CT in detection of recurrence and metastasis of colorectal cancer. World J Gastroenterol, 2007; 13(37): 5025-29. 